\title{
Culture and odor categorization: agreement between cultures depends upon the odors
}

\author{
C. Chrea ${ }^{\text {a,* }}$, D. Valentin ${ }^{\text {a,b }}$, C. Sulmont-Rossé ${ }^{\text {c }}$, H. Ly Mai ${ }^{b}$, D. Hoang Nguyen ${ }^{\text {d, }}$ \\ H. Abdi ${ }^{\mathrm{e}}$ \\ ${ }^{a}$ Centre Européen des Sciences du Goût, 15 rue Hugues Picardet, 21000 Dijon, France \\ ${ }^{\mathrm{b}}$ University of Bourgogne, Esplanade Erasme, 21000 Dijon, France \\ ${ }^{\mathrm{c}}$ Unité Mixte de Recherche sur les Arômes, INRA-ENESAD, 17 Rue Sully, BP 86510, 21065 Dijon Cedex, France \\ ${ }^{\mathrm{d}}$ University of Technology of Ho Chi Minh City, 268 Ly Thuong Kiet street, D10, HCM City, Viet Nam \\ ${ }^{\mathrm{e}}$ The University of Texas at Dallas, 2601 North Floyd Road, Richardson, TX 75080, USA
}

Received 16 July 2003; received in revised form 21 October 2003; accepted 24 October 2003

Available online 6 December 2003

\begin{abstract}
This study evaluated the effect of culture on the relationship between psychological dimensions underlying odor perception and odor categorization. In a first experiment, French, Vietnamese and American participants rated several perceptual dimensions of everyday odorants, and sorted these odorants on the basis of their similarity. Results showed that the three groups of participants differed in their perceptual judgments but agreed in categorizing the odors into four consensual groups (floral, sweet, bad, and nature). Three dimensions - pleasantness, edibility, cosmetic acceptability - discriminated these groups in the same way in the three countries. In a second experiment, the participants sorted only fruit and flower odors to evaluate whether a consensus emerges at a finer level. Results showed that French and American participants clearly separated fruit from flower odors whereas this separation was nonexistent for Vietnamese participants. This difference could arise from cultural differences in odor functions.
\end{abstract}

(C) 2003 Elsevier Ltd. All rights reserved.

Keywords: Odor; Culture; Categorization; Representation

\section{Introduction}

Cross-cultural studies in odor perception have been carried out from two points of view: To understand cultural differences in food acceptance and preference (Prescott \& Bell, 1995; Yeh et al., 1998) and to evaluate the effect of individual experience and knowledge of odors on odor perception in order to understand how olfactory stimuli influence physiology and behavior (Hudson \& Distel, 2002). However, these studies have not specifically addressed the fundamental issue of the origins of these cultural differences in odor perception and preferences. These studies can be sorted in four main types according to the issue they address.

\footnotetext{
${ }^{*}$ Corresponding author. Fax: +33-3-80-68-16-01.

E-mail address: chrea@cesg.cnrs.fr (C. Chrea).
}

\subsection{Effect of culture on perceptual judgments}

A first type of cross-cultural studies has investigated the effect of culture on several dimensions such as familiarity, pleasantness, intensity, and edibility for everyday odors (Aubaile Sallenave, 2000; AyabeKanamura et al., 1998; Distel et al., 1999; Pangborn, Guinard, \& Davis, 1988; Song \& Bell, 1998; Wysocki, Pierce, \& Gilbert, 1991). The objectives of these studies were twofold: (a) to evaluate the stability of any relationship between those perceptual dimensions across different cultures and (b) to quantify the perceptual differences between cultures. Globally, results showed that, in all countries, familiarity was correlated with both intensity and pleasantness: Whatever the country, participants perceived and liked better the odors with which they were more familiar. Additionally, differences between cultures appeared on pleasantness, and to a smaller extend, on familiarity, edibility, and intensity ratings. These differences concerned mostly food odors 
and were higher for odors specific to a given culture than odors common to all cultures. In contrast, a cultural consensus to judge odors such as body odors, odors of decomposed biological materials, or fecal odors as unpleasant has been reported in studies involving different cultures from several continents (Dilks, Dalton, \& Beauchamp, 1999; Schaal et al., 1997; Schleidt, Hold, \& Attili, 1981; Wysocki et al., 1991).

\subsection{Effect of culture on odor sensitivity}

To the best of our knowledge, the only recent study investigating the effect of culture on odor sensitivity was carried out by Hübener, Laska, Kobayakawa, and Saito (in press) with Japanese and German people. These authors found that Japanese people have a lower detection threshold for two of the different tested odorants: an odorant characteristic of the Japanese culture (Japanese ink) and an odorant characteristic of the German culture (aniseed). A remaining question is to know which of the genetically, developmental, physiological, psychological, or cultural factors are the more relevant to gain a better understanding of these results.

\subsection{Effect of culture on odor identification}

Some authors have investigated the effect of culture on the ability to identify or describe everyday odors. Doty, Applebaum, Zusho, and Settle (1985) showed that American Korean participants outperformed Caucasian and African American participants, who, in turn, outperformed Native Japanese participants in an identification task using the standardized test UPSIT. Although the authors could not explain these differences by strictly cultural factors, they observed that familiarity with the tested odorants was a factor of influence on identification performance. This finding is in agreement with the results of Rabin and Cain (1984) who found that odors are more accurately identified when they are familiar. Aubaile Sallenave (2000) further added that odor descriptions depend on the function attributed to the odor. For instance, the odor of apple is easily associated to cosmetic products in cultures where this odor is used to scent shower gel or shampoo but not in more traditional cultures where cosmetic products are not scented with this type of odor.

\subsection{Effect of culture on odor representation}

A last type of studies has investigated the effect of culture on olfactory mental representation. Schleidt, Neumann, and Morishita (1988) asked German and Japanese participants to spontaneously recall odor memories and rate pleasantness of each odor memory. The authors then classified empirically the answers of participants in five similar lexical categories. The results showed that the sizes of categories and the number of pleasant and unpleasant odors within these categories were comparable across the two cultures. The authors also found some differences on the nature of the odors within some categories that may reflect differences in sleeping and housing environment. In a study carried out with odors per se, Ueno (1993) showed that Japanese and Sherpa participants agreed on the way to sort 20 artificial Japanese aromas on the basis of their perceptual similarity, with the exception that the Japanese classification revealed a "fishy" category that did not emerge in the Sherpa classification. The authors hypothesized that this result could reflect differences in food habits because Sherpa people are not used to eating fish.

In summary, all the studies presented above clearly revealed that cultural differences and similarities exist in both judgment for several psychological dimensions and odor representation. Thus it is worth evaluating further the relation between psychological dimensions underlying odor perception and odor representation in order to evaluate (1) whether people of different cultures organize their knowledge of odors on the basis of common psychological dimensions and (2) whether cultural differences in these dimensions may explain differences in odor representation across cultures.

To address theses issues, we carried out a cross-cultural study with American, French, and Vietnamese participants. We designed several experiments including a rating task and a sorting task. The aim of the rating task was to evaluate the dimensions underlying odor perception. Six perceptual dimensions were studied: familiarity, pleasantness, intensity, saliency, edibility and cosmetic acceptability. The sorting task was designed to evaluate if people of different cultures categorize odors in similar perceptual categories. The aim of the first study presented in this paper was to evaluate whether the odor space resulting from the sorting task may be predicted by the several perceptual dimensions in the three cultures.

\section{Study 1}

\subsection{Methods}

\subsubsection{Participants}

Three groups of sixty students for the rating task and three groups of 30 students for the sorting task were recruited respectively from The University of Texas at Dallas in the USA, from the University of Bourgogne at Dijon in France, and from the Polytechnic Institutes of Hanoi and Danang in Vietnam. All groups were comparable in gender and age distributions, both across the tasks and across the countries (Table 1). All the participants were born and raised in the studied countries. 
Table 1

Sex repartition and mean age with standard deviation for study 1 and 2

\begin{tabular}{|c|c|c|c|c|c|c|}
\hline & \multicolumn{2}{|l|}{ France } & \multicolumn{2}{|l|}{ The USA } & \multicolumn{2}{|l|}{ Vietnam } \\
\hline & Females & Males & Females & Males & Females & Males \\
\hline Study 1 & $21(20.8 \pm 1.7)$ & $9(22.1 \pm 1.83)$ & $23(23.4 \pm 3.2)$ & $7(23.3 \pm 3.4)$ & $20(22.5 \pm 0.9)$ & $10(22.5 \pm 1.5)$ \\
\hline Study 2 & $13(25.3 \pm 2.63)$ & $7(29.1 \pm 3.93)$ & $16(23.7 \pm .52)$ & $4(27.5 \pm 8.22)$ & $14(22.3 \pm 1.54)$ & $6(22.3 \pm 1.86)$ \\
\hline
\end{tabular}

They were naive to the purpose of the experiment and were not familiar with olfactory testing.

\subsubsection{Stimuli}

2.1.2.1. Rating task. Fifty-six odorants were selected from an initial set of 83 odor samples provided by Sentosphère (59, bd du Général Valin, 75015 Paris). A pilot study, run only in France, consisted in a lexical sorting task. The goal of this pilot study was to obtain an a priori lexically structured space and to select a stimulus set from the large choice of odors made by Sentosphère. Twelve French subjects $(2$ males and 10 females; age range 21-48) were asked to sort freely the 83 odor labels of Sentosphère in as many groups as they wanted and to label each group. Six lexical clusters emerged from these data: fruit, flower, spice, pharmacy, chemicals, breakfast, animal, musty. Two-third of the samples of each cluster were selected to obtain a set of 56 odorants. The set of odorants included some odorants thought to be typical of France, America, and Vietnam, and some thought to be common to all cultures (Table 2). Odorants were presented as microencapsulated odorants in $2 \mathrm{~cm}$ high punched plastic flasks randomly coded by a 3 -digit number.

2.1.2.2. Sorting task. To avoid too much olfactory fatigue for the participants, only 40 odorants from the

Table 2

List of odorants for study 1 and 2

\begin{tabular}{|c|c|c|}
\hline Amber $^{\mathrm{a}}$ & Hazelnut $^{\mathrm{a}}$ & Peanut $^{\mathrm{a}}$ \\
\hline Anise $^{a}$ & Honey $^{\mathrm{a}}$ & Pepper \\
\hline Apricot ${ }^{\mathrm{a}, \mathrm{b}}$ & Honeysuckle $^{\mathrm{b}}$ & Pine $^{\mathrm{a}}$ \\
\hline Banana $^{b}$ & Jasmine $^{\mathrm{a}, \mathrm{b}}$ & Pineapple ${ }^{\mathrm{a}, \mathrm{b}}$ \\
\hline Blackcurrant $^{\mathrm{a}, \mathrm{b}}$ & Lavender ${ }^{\mathrm{a}, \mathrm{b}}$ & $\operatorname{Rose}^{\mathrm{a}, \mathrm{b}}$ \\
\hline Butter $^{\mathrm{a}}$ & Leather $^{\mathrm{a}}$ & Soap $^{\mathrm{a}}$ \\
\hline Camphor & Lilac ${ }^{\mathrm{b}}$ & Strawberry ${ }^{\mathrm{a}, \mathrm{b}}$ \\
\hline Cat pee ${ }^{a}$ & Lily of the Valley ${ }^{b}$ & Sulfur \\
\hline Chocolate & Litchi $^{\mathrm{b}}$ & $\mathrm{Tea}^{\mathrm{a}}$ \\
\hline Cinnamon $^{\mathrm{a}}$ & Mango $^{\mathrm{a}, \mathrm{b}}$ & Truffle $^{a}$ \\
\hline Civet $^{\mathrm{a}}$ & Melon $^{\mathrm{a}, \mathrm{b}}$ & Turpentine \\
\hline Clove $^{a}$ & Milk $^{\mathrm{a}}$ & Vanilla $^{\mathrm{a}}$ \\
\hline Coconut $^{\mathrm{b}}$ & Mint & Violet $^{\mathrm{a}, \mathrm{b}}$ \\
\hline Cookies $^{\mathrm{a}}$ & Moth ball ${ }^{\mathrm{a}}$ & Walnut $^{\mathrm{a}}$ \\
\hline Detergent $^{\mathrm{a}}$ & Moldy $^{\mathrm{a}}$ & Wild beast \\
\hline Earth & Mushroom $^{\mathrm{a}}$ & Wintergreen $^{\mathrm{a}}$ \\
\hline Eucalyptus $^{\mathrm{a}}$ & Musk $^{\mathrm{a}}$ & Woody ${ }^{\mathrm{a}}$ \\
\hline Garlic & Nutmeg ${ }^{\mathrm{a}}$ & Ylang ylang ${ }^{\mathrm{b}}$ \\
\hline Ginger $^{\mathrm{a}}$ & Orange blossom ${ }^{\mathrm{a}, \mathrm{b}}$ & \\
\hline
\end{tabular}

${ }^{\mathrm{a}}$ The 40 odorants used in the sorting task of study 1 .

${ }^{\mathrm{b}}$ The 18 odorants used in the sorting task of study 2 . original set were kept for the sorting task. They were selected on the basis of the highest differences in familiarity rating between the three cultures in order to have both familiar and unfamiliar odorants for each culture. The flasks were randomly coded by a 2 -digit number ranging from 1 to 40 .

\subsubsection{Procedure}

2.1.3.1. Rating task. Because of the number of perceptual judgments we studied, we divided the rating task into two subtasks and each participant performed each one of these subtasks. For each of the subtasks, participants were presented with the 56 odorants in a randomized order. After smelling an odorant, they were asked to answer several questions on 7-point scales labeled at each end of the scale. In the first subtask, participants answered six questions: one question on familiarity (how familiar is this odor? where ' 1 ' is "not familiar at all" and ' 7 ' is "very familiar"); one question on intensity (how intense is this odor? where ' 1 ' is "very weak" and ' 7 ' is "very strong"); two questions on hedonic value (how pleasant is this smell? where ' 1 ' is "not pleasant at all" and '7' is "very pleasant" and do you like this odor? where '1' is "not at all" and '7' is "a lot"); two questions on saliency (how easy it would be to memorize this odor? and how easy it would be to distinguish this odor from a set of odors? where ' 1 ' is "very difficult" and '7' is "very easy"). In a second subtask, participants answered two questions: one question on edibility (would you accept to eat a food with this odor? where ' 1 ' is "not agree at all" and ' 7 ' is "strongly agree") and one question on cosmetic acceptability (would you accept to wear a perfume or a cosmetic product with this odor?where ' 1 ' is "not agree at all" and ' 7 ' is "strongly agree").

For each of the subtasks, the question presentation order was counterbalanced across participants, but for a given participant, this presentation order was the same for all odors. The participants' answers were recorded by an APPLE computer running the PsyScope data acquisition software (Cohen, Whinney, Flatt, \& Provost, 1993). For each odorant, questions appeared on separate screens to obtain answers that were as independent as possible.

2.1.3.2. Sorting task. The participants were presented with the 40 odorant stimuli in a randomized order. The participants were asked to sort the 40 stimuli into 
groups on the basis of odor similarity. They were allowed to form as many groups as they wanted, and to put as many odors as they wished in each group. When they had finished this task, participants were asked to provide a few descriptors for each of the groups they had formed.

\subsubsection{Experimental condition}

For each task, the presentation order of stimuli was counterbalanced across participants. Participants were instructed to smell the odorants by breathing normally, without sniffing. A break of $15 \mathrm{~s}$ was imposed on the participants after each odorant in order to reduce olfactory adaptation. If a participant perceived no odor when smelling an odorant, he or she did not perform the task for this odorant.

\subsection{Data analysis}

All statistical analyses were conducted using SAS/ STAT $^{\circledR}$ (SAS, 1989). As we were interested in correlating the result of the sorting task and the rating task, all the analyses presented here were performed only on the set of 40 odorants used in the sorting task.

\subsubsection{Rating task}

Mean rating scores were calculated by odor for the eight judgments in all three cultures. The $1 \%$ of cases in France and $0.6 \%$ in the USA in which participants did not perceive a particular odorant were excluded from the data. The correlation between all perceptual judgments was calculated using Pearson's correlation coefficients. A two-ways analysis of variance with culture as a between-participant independent variable and odor as a within-participant independent variable was performed for each perceptual judgment. When the odor by culture interaction was significant, a Duncan test was performed. An alpha value of 0.01 was taken as the significance level throughout.

\subsubsection{Sorting task}

For each culture, we started by deriving pairwise similarity estimates by counting the number of times two odors were sorted into the same group. The three resulting co-occurrence matrices were submitted to a multidimensional scaling (MDS) analysis using the nonparametric Alternating Least-Square sCALing (ALSCAL) algorithm. This analysis was completed by a Hierarchical Cluster Analysis (HCA).

\subsection{Results}

\subsubsection{Rating task}

Table 3 reports the correlation coefficients between the eight perceptual judgments. This table shows that familiarity is correlated with all the other judgments except cosmetic acceptability in Vietnam. In the same way, pleasantness and liking are correlated with distinctiveness, memory, cosmetic acceptability and edibility in all three cultures except with memory in Vietnam. Furthermore, in all three cultures, pleasantness is strongly correlated with liking and distinctiveness is strongly correlated with memory. Thus, we decided to average pleasantness and liking variables in only one variable called pleasantness. In the same way, we averaged distinctiveness and memory variables in one variable called saliency.

We performed the two-way analysis of variance on six variables: familiarity, intensity, pleasantness, saliency, cosmetic acceptability and edibility. Results show a significant main effect of culture for all perceptual judgments except cosmetic acceptability: familiarity $\left(F_{(2,87)}=12.50, \quad P<0.0001\right)$, intensity $\left(F_{(2,87)}=29.03\right.$, $P<0.0001)$, pleasantness $\left(F_{(2,87)}=90.49, P<0.0001\right)$, saliency $\quad\left(F_{(2,87)}=12.33, \quad P<0.01 \quad\right.$ and edibility $\left(F_{(2,87)}=13.98, \quad P<0.0001\right)$. Vietnamese participants gave higher ratings compared to the other cultural groups for all these dimensions except for intensity for which they gave a lower rating. American and French ratings did not differ significantly except for saliency and edibility; the American ratings were higher than the French ones for saliency whereas French ratings were higher than the American ones for edibility. These results are globally in agreement with previous cross-cultural studies. Moreover, the two-way analysis of variance indicates that there is a highly significant effect of odor by culture interaction for all the perceptual judgments. This high effect, however, might be due to the large number of degree of freedom. Nonetheless an analysis of the effect of culture is higher for odorants specific to a culture than for odorants common to all three cultures. For instance, in France, lavender was judged as more familiar and more acceptable as a cosmetic, while cinnamon was perceived as less familiar, pleasant, intense, salient and edible compared to the USA and Vietnam. In Vietnam, ginger and mango were perceived as more familiar, more pleasant, more salient and more edible, whereas anise was perceived as less familiar, less pleasant, less salient, less intense and less edible compared to the USA and France. Wintergreen was perceived as more pleasant in the USA. Likewise, apricot, strawberry, pineapple, coconut, cookies, violet and amber were perceived similarly for all perceptual judgments across the three cultures.

\subsubsection{Sorting task}

For all three cultures, three dimensions were selected as the most appropriate MDS solution; the stress values were respectively 0.16 for France, 0.16 for the USA, and 0.18 for Vietnam (see Fig. 1). From the HCA, we found 
Table 3

Matrix of Pearson correlations between the eight perceptual judgments

\begin{tabular}{|c|c|c|c|c|c|c|c|c|}
\hline & Familiarity & Intensity & Pleasantness & Liking & Distinctiveness & Memory & Cosmetic & Edibility \\
\hline \multicolumn{9}{|l|}{ France } \\
\hline Familiarity & - & $0.48^{* *}$ & $0.73^{* * *}$ & $0.78^{* * *}$ & $0.78^{* * *}$ & $0.89^{* * *}$ & $0.45^{* *}$ & $0.59^{* * *}$ \\
\hline Intensity & & - & $0.08 \mathrm{~ns}$ & $0.16 \mathrm{~ns}$ & $0.82^{* * *}$ & $0.69^{* * *}$ & $-0.08 \mathrm{~ns}$ & $0.23 \mathrm{~ns}$ \\
\hline Pleasantness & & & - & $\mathbf{0 . 9 8}^{* * *}$ & $0.38^{*}$ & $0.51^{* * *}$ & $0.83^{* * *}$ & $0.69^{* * *}$ \\
\hline Liking & & & & - & $0.47^{* *}$ & $0.69^{* * *}$ & $0.78^{* * *}$ & $0.74^{* * *}$ \\
\hline Distinctiveness & & & & & - & $0.93^{* * *}$ & $0.14 \mathrm{~ns}$ & $0.45 \mathrm{~ns}$ \\
\hline Memory & & & & & & - & $0.25 \mathrm{~ns}$ & $0.51^{* * *}$ \\
\hline Cosmetic & & & & & & & - & $0.37^{*}$ \\
\hline Edibility & & & & & & & & - \\
\hline \multicolumn{9}{|l|}{ The USA } \\
\hline Familiarity & - & $0.39^{*}$ & $0.80^{* * *}$ & $0.81^{* * *}$ & $0.81^{* * *}$ & $0.82^{* * *}$ & $0.45^{* *}$ & $0.65^{* * *}$ \\
\hline Intensity & & - & $0.02 \mathrm{~ns}$ & $0.02 \mathrm{~ns}$ & $0.74^{* * *}$ & $0.74^{* * *}$ & $-.24 \mathrm{~ns}$ & $0.18 \mathrm{~ns}$ \\
\hline Pleasantness & & & - & $0.99^{* * *}$ & $0.45^{* *}$ & $0.47^{* *}$ & $0.78^{* * *}$ & $0.65^{* * *}$ \\
\hline Liking & & & & - & $0.47^{* *}$ & $0.49^{* *}$ & $0.78^{* * *}$ & $0.66^{* * *}$ \\
\hline Distinctiveness & & & & & - & $0.98^{* * *}$ & $0.11 \mathrm{~ns}$ & $0.53^{* * *}$ \\
\hline Memory & & & & & & - & $0.15 \mathrm{~ns}$ & $0.49^{* *}$ \\
\hline Cosmetic & & & & & & & - & $0.21 \mathrm{~ns}$ \\
\hline Edibility & & & & & & & & - \\
\hline \multicolumn{9}{|l|}{ Vietnam } \\
\hline Familiarity & - & $0.31^{*}$ & $0.55^{* * *}$ & $0.51^{* * *}$ & $0.80^{* * *}$ & $0.76^{* * *}$ & $0.31 \mathrm{~ns}$ & $0.36^{*}$ \\
\hline Intensity & & - & $0.32^{*}$ & $0.33^{*}$ & $0.36^{*}$ & 0.28 & $0.41^{* *}$ & $0.51^{* * *}$ \\
\hline Pleasantness & & & - & $0.95^{* * *}$ & $0.40^{*}$ & $0.28 \mathrm{~ns}$ & $0.42^{* *}$ & $0.53^{* * *}$ \\
\hline Liking & & & & - & $0.36^{*}$ & $0.28 \mathrm{~ns}$ & $0.42^{* *}$ & $0.51^{* * *}$ \\
\hline Distinctiveness & & & & & - & $0.86^{* * *}$ & $0.15 \mathrm{~ns}$ & $0.37^{*}$ \\
\hline Memory & & & & & & - & $0.05 \mathrm{~ns}$ & $0.26 \mathrm{~ns}$ \\
\hline Cosmetic & & & & & & & - & $0.24 \mathrm{~ns}$ \\
\hline Edibility & & & & & & & & - \\
\hline
\end{tabular}

ns, not significant.

${ }^{*} P<0.05$.

${ }^{* *} P<0.01$.

${ }^{* * *} P<0.001$

that we could select five clusters in France and four clusters in Vietnam and the USA. For each culture, those clusters are indicated in Fig. 1 by different symbols.

Overall, Fig. 1 shows that, despite some obvious differences, the odor spaces obtained for the three cultural groups are similar. Four similar clusters seem to emerge from all three cultural configurations. A first cluster (we labeled "floral") includes odors of flowers, cleaning products and cosmetics and was described with these terms by the three cultural groups. A second cluster (we labeled "sweet") includes fruit odors and some odors such as vanilla and cookies and was described by the three cultural groups as sweet, candy and fruity odors. A third cluster (we labeled "bad") includes odors of animals, mustiness, and nuts and was described by the three cultural groups as bad and moldy odors. A fourth cluster (we labeled "nature") includes tea odor, some musty and spice odors (e.g. woody, nutmeg) and some flower odors. This cluster was described as outdoor and spicy odors by the American group, as spicy, peppery, natural odors by the French group and as plant, moldy, floral odors by the Vietnamese group. An additional fifth cluster (we labeled "medicine") was observed for the French group. It includes odors of anise, cinnamon, eucalyptus, mango and wintergreen and was described as spicy, fresh, medicine odors.

Aside from this cultural agreement, some differences are also noteworthy and may be due to cultural differences in food and household habits. For instance, only the American participants sorted the odors of anise, cinnamon and wintergreen, which are frequently used as candy or soda flavors in the USA, in the "sweet" cluster. These odors were sorted in the "medicine" cluster by the French participants and were mostly associated to odors of medicine to cure cold. In Vietnam, these odors, found in the composition of traditional medicine such as "tiger balm" were sorted in the "floral" cluster which was also described as "traditional medicine" odors by the Vietnamese participants. Another difference emerges for mango odor. This odor was sorted in the "sweet" cluster in Vietnam with the other fruits whereas it was sorted in the "floral" cluster in the USA and in the "medicine" cluster in France. We can suppose that mango, being an Asian fruit, may have a more familiar odor to Vietnamese people and thus be sorted with other fruits by Vietnamese participants. 

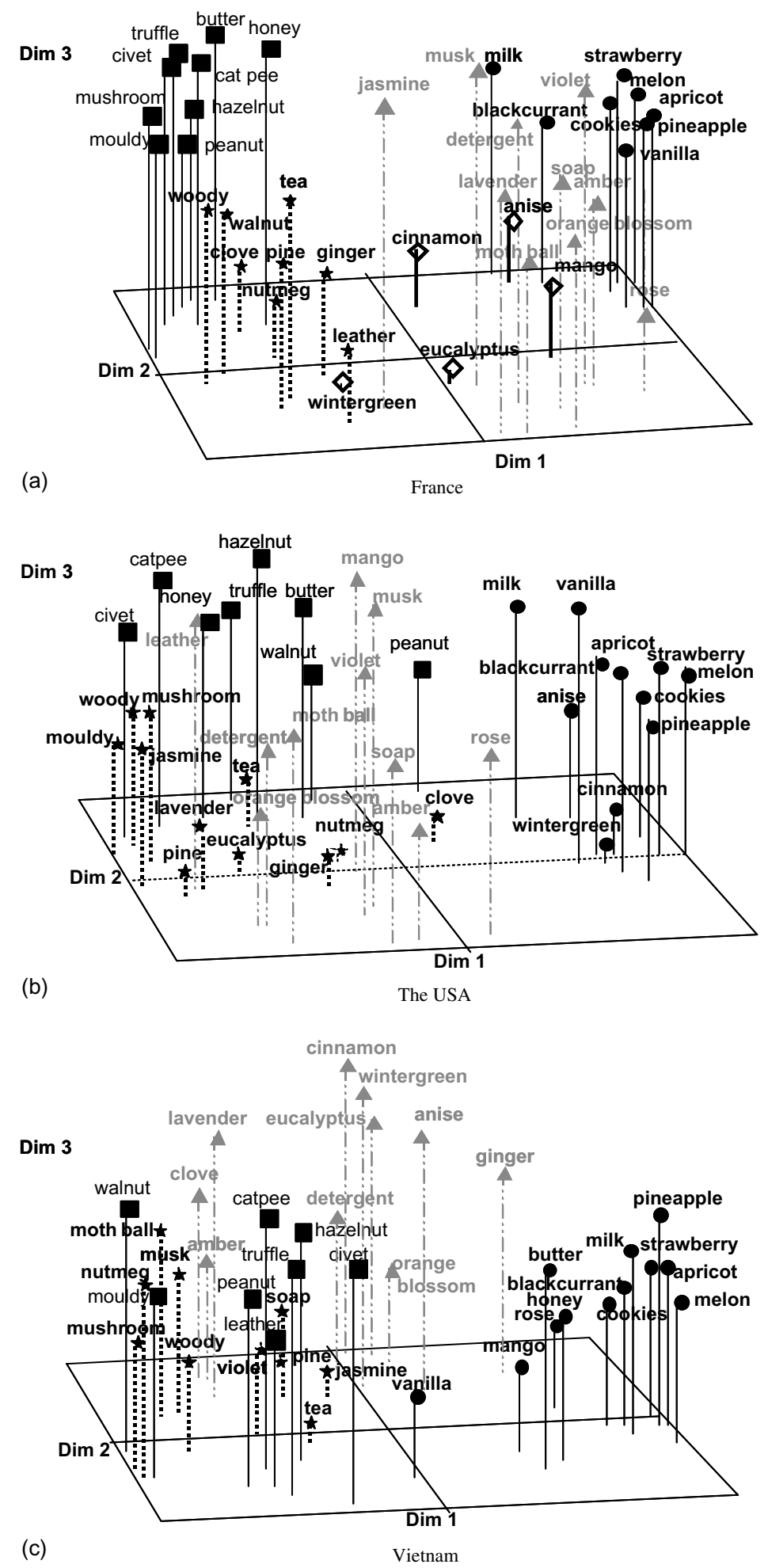

Fig. 1. Three-dimensional solutions from odor sorting made by participants of the three cultures (Dim denotes dimension). The different clusters are marked by different symbols. ( $\Delta$ ) Floral cluster; $(\bullet)$ sweet cluster; $(\mathbf{\square})$ bad cluster; $(\star)$ nature cluster; $(\diamond)$ medicine cluster.

\subsubsection{Relation between the sorting task and the rating task}

To determine the perceptual dimensions underlying the odor category structure, a linear discriminant anal- ysis was performed for each culture using the six perceptual variables (i.e., familiarity, intensity, pleasantness, saliency, edibility and cosmetic acceptability as predictors of membership in the clusters yielded by the HCAs. 
The discriminant analysis is significant for all three cultures, $\quad\left(F_{(24,106)}=6.48, \quad P<0.0001\right.$ for France, $F_{(18,88)}=6.43, P<0.0001$ for the USA, $F_{(18,88)}=3.94$, $P<0.0001$ for Vietnam). Two significant discriminant functions maximize the discrimination of the 40 odors for all three cultures. These two linear discriminant functions account together for $93 \%$ of the variance for France and the USA and $95 \%$ for Vietnam. The matrix of correlations between the predictors and the first two discriminant functions, as seen in Table 4 , suggests that edibility and pleasantness have the largest loading on the first axis for France and Vietnam, while cosmetic acceptability has the largest loading for the USA. Cosmetic acceptability has the largest loading on the second axis for France and Vietnam whereas pleasantness and edibility load strongly on the second axis for the USA. These results suggest that three common dimensions, edibility, pleasantness, and cosmetic acceptability underlie cluster membership for the three groups of participants. As shown in Fig. 2, in all three cultures, edibility and pleasantness seem to separate nature and bad clusters from floral and sweet clusters (i.e., axis 1 for France and Vietnam and axis 2 for the USA). Cosmetic acceptability (i.e., axis 2 for France and Vietnam and axis 1 for the USA) discriminates sweet from floral cluster with the other clusters falling between these two clusters for France and the USA. These dimensions globally discriminate clusters in a similar way for the three cultures. However, according to the discriminant analysis and as reflected in the sorting task, there is some difference among the cultures: French and Vietnamese participants gave more weight to edibility and pleasantness, whereas American participants gave more weight to cosmetic acceptability.

\subsection{Discussion}

These results show both differences and similarities in the way odors are perceived in different cultures. On the one hand, differences between the three groups of participants exist on judgment for several perceptual dimensions and may be due mainly to cultural

Table 4

Matrix of correlations between the six perceptual variables as predictors and the first two discriminant functions

\begin{tabular}{|c|c|c|c|c|c|c|}
\hline \multirow[t]{2}{*}{ Predictor variable } & \multicolumn{2}{|l|}{ France } & \multicolumn{2}{|c|}{ The USA } & \multicolumn{2}{|c|}{ Vietnam } \\
\hline & $\overline{F 1}$ & $F 2$ & $\overline{F 1}$ & $F 2$ & $F 1$ & $F 2$ \\
\hline Familiarity & 0.29 & 0.13 & 0.40 & 0.54 & 0.50 & 0.12 \\
\hline Intensity & -0.05 & 0.05 & 0.25 & -0.13 & 0.05 & 0.06 \\
\hline Pleasantness & 0.62 & 0.30 & 0.31 & 0.93 & 0.54 & 0.24 \\
\hline Saliency & 0.17 & 0.16 & 0.44 & 0.20 & 0.33 & 0.09 \\
\hline Edibility & 0.87 & -0.21 & -0.09 & 0.89 & 0.75 & -0.25 \\
\hline Cosmetic & 0.33 & 0.79 & 0.81 & 0.37 & 0.43 & 0.68 \\
\hline
\end{tabular}
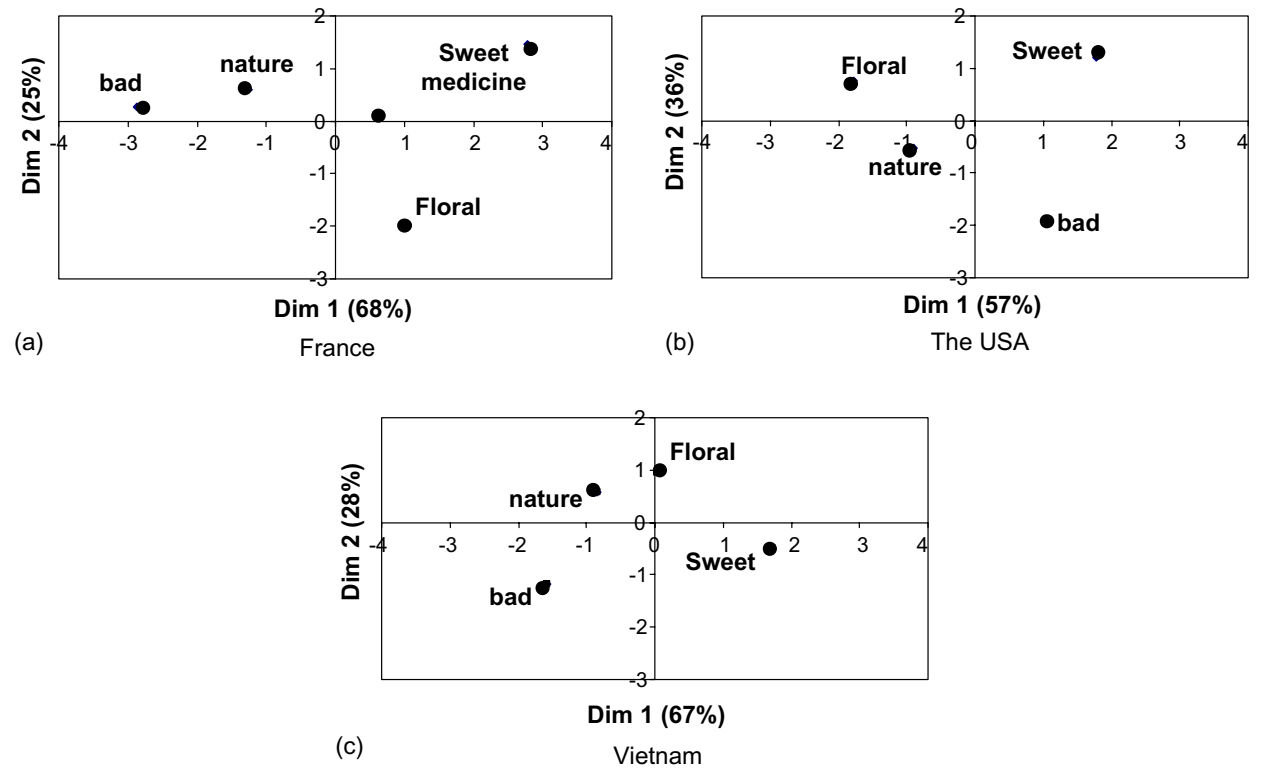

Fig. 2. Plots of the cluster centroids on the first two discriminant functions derived from six perceptual variables in the three cultures. 
differences in food and household habits (AyabeKanamura et al., 1998). On the other hand a consensus between the three groups of participants seems to emerge in the way to categorize odors. Furthermore this consensus is supported by the fact that the three common dimensions of edibility, pleasantness and cosmetic acceptability underlie the odor representation structure for the three cultural groups. These results confirm previous studies that used the MDS method to evaluate the relevant dimensions underlying odor perception (Berglund, Berglund, Engen, \& Ekman, 1973; Schiffman, Robinson, \& Erickson, 1977; Woskow, 1968). These authors showed that the hedonic dimension was the most relevant and separated the overall odor space in odors that people like and odors that people dislike. Our results add further information and are in part in agreement with a recent study carried out by Sugiyama, Ayabe-Kanamura, \& Kikuchi (2002). Using a similarity judgment task and a MDS analysis, these authors found that the edibility dimension was correlated with one of the dimensions of the MDS. These findings support strongly the hypothesis that odor representation is not based on a unique continuum, but is a manifold of several psychological dimensions (Holley, 2001). Furthermore, a remaining question is to know to what extent this consensus may be consistent at a finer level with a reduced set of odors. Lawless (1989) argued that a limited section of odor space may minimize the importance of the hedonic dimension. With a reduced odor space composed of citrus and woody odors, he showed a strong structure of the odor space with a dichotomy woody/citrus. It is worth evaluating whether the crosscultural consensus we found for a large set of various odors still exist in a smaller odor space.

To answer this question, we decided, following Lawless (1989), to focus on two general categories of odors, namely fruit and flower. Our choice was made according to the results of the MDS analysis reported previously. As shown in Fig. 1, some obvious cultural differences emerge concerning the boundaries of the "floral" and "sweet" clusters. Indeed, these clusters are very close in France whereas they are far apart in the USA and Vietnam. These two clusters seem relevant for investigating further the effect of culture on odor representation at a finer level. Thus we designed a second experiment based on a sorting task of fruit and flower odorants. In contrast to Lawless's (1989) which included two experimental conditions (i.e. an unlimited number of groups and a restricted one to two groups), participants performed the task only in the restricted condition of sorting the odors in only two groups in order (1) to determine whether people of different cultures categorize naturally fruit odorants on a side and flower odorants on the other side, and (2) to evaluate the stability of the boundaries of these categories across the three cultures.

\section{Study 2}

\subsection{Method}

\subsubsection{Participants}

Twenty participants from each culture were recruited from The University of Texas at Dallas in the USA, the University of Bourgogne at Dijon in France and the Polytechnic Institute of Danang in Vietnam. Groups were comparable in gender and age distribution across the countries (Table 1). The participants were born and raised in the country of the experiment. All were naive to the purpose of the experiment and not familiar with olfactory testing.

\subsubsection{Stimuli}

Because the number of fruit and flower odorants used in the previous sorting task was too small to constitute consistent categories, we added four fruit and four flower odorants to the previous ones. Thus, nine fruit odorants and nine flower odorants were selected from the original set of 56 odorants provided by Sentosphère (see Table 2). They were randomly coded by a 2-digit number ranging from 1 to 18 .

\subsubsection{Procedure}

Stimuli were presented in a random order in front of the participants. Participants were asked to smell the stimuli and sort them in only two groups on the basis of their perceptual similarity. No indication was given to the participants about the criterion they may use to classify the odors and each group could contain as many stimuli as the participants wished. After completion of the sorting task, participants were asked to provide a few words to describe each of the two groups they had formed. The experimental conditions were similar to the previous sorting task.

\subsection{Data analysis}

To evaluate the similarity between the fruit and flower odorants, we first derived pairwise similarity estimates in each culture by counting the number of times two odors were sorted into the same group. The three resulting distance matrices were submitted to an additive tree analysis (Abdi, 1990). This multivariate analysis is used to represent objects as "leaves" of a tree, so that the distance on a tree between two leaves reflects the similarity between the two objects. This method was preferred to the traditional MDS because of its better approximation of similarity distances and because it is particularly adapted for a small set of stimuli. 


\subsection{Results}

Fig. 3 shows the additive trees for the three cultures. These representations account for $92 \%$ of the variance in France, $74 \%$ in the USA and $47 \%$ in Vietnam. A first point to note is the remarkable difference between the Vietnamese additive tree on one side and the American and the French ones on the other side. Indeed, the French and the American trees show a clear separation between the fruit and flower odorants. The descriptors given by American and French participants strongly support this observation: the fruit cluster is described as sweet and fruity; the floral cluster is described as floral and cosmetic. Moreover some odors are misclassified in the same way in both countries: mango and litchi seem to be categorized with the flowers; apricot for both countries and blackcurrant and rose for the USA fall between the two categories. However, a difference emerges between the French and the American trees: the French tree is strongly structured in two subclusters whereas the American one reveals three subclusters, with a subdistinction for flowers between pleasant and unpleasant flower odorants. This observation reflects simply the fact that the agreement between participants was stronger in France than in the USA. In contrary, the Vietnamese tree is structured in five subclusters that do not refer clearly to the quality of the odorants but more to the intensity and the pleasantness. These five subclusters can be separated into two unpleasant subclusters "floral-unpleasant" and "strong-unpleasant" on the right side and three pleasant subclusters "sweet-pleas- ant", "fruity-pleasant", and "strong-fragrant-pleasant" on the left side.

The results and conclusions from the additive tree analysis were confirmed by classical MDS analyses. According to the stress value profile, a two-dimensional configuration was selected for all three cultures (Fig. 4). Fig. 4 shows that fruit and flower odorants are clearly separated in France and the USA while they are widely spread in Vietnam.

\subsection{Discussion}

These results show that the consensus emerging at a macro structural level between the three cultures is consistent for only two cultures at a microstructural level. French and American participants tended to categorize fruit and flower odors in two general categories whereas Vietnamese participants categorized them mostly according to the intensity and the hedonics of the odorants. This difference may come from cultural differences on odor knowledge. Additional data showed that in a free identification task, American and French participants identified the odors more precisely than Vietnamese participants and moreover more often with the label given by Sentosphère for French participants (Ly May, 2001). Rabin \& Cain (1984) have shown that odor recognition performance is better when the participant can identify the odor. In the case of a sorting task, we can suppose that identification helps participants to find some lexical criterion to form their groups. In agreement with this hypothesis, Chastrette,

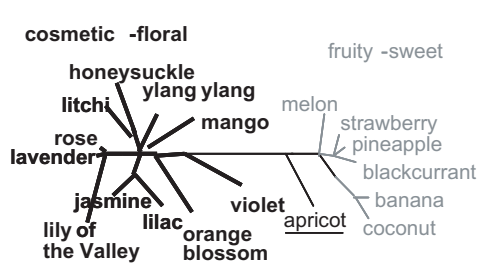

France

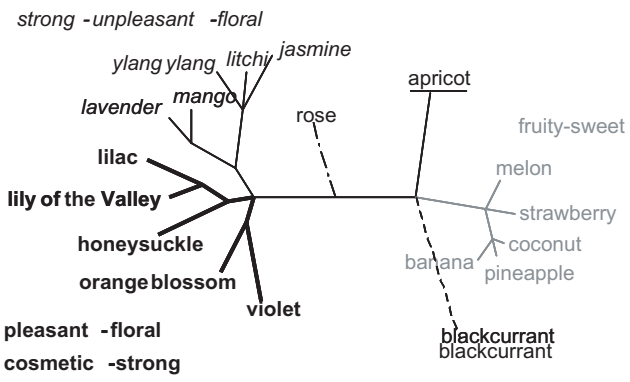

the USA

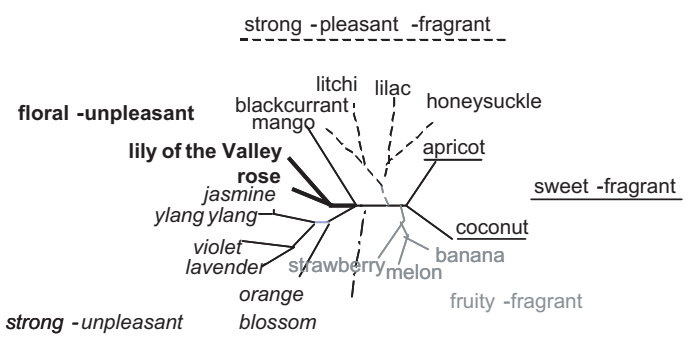

Vietnam

Fig. 3. Additive tree representations for the three cultures. 

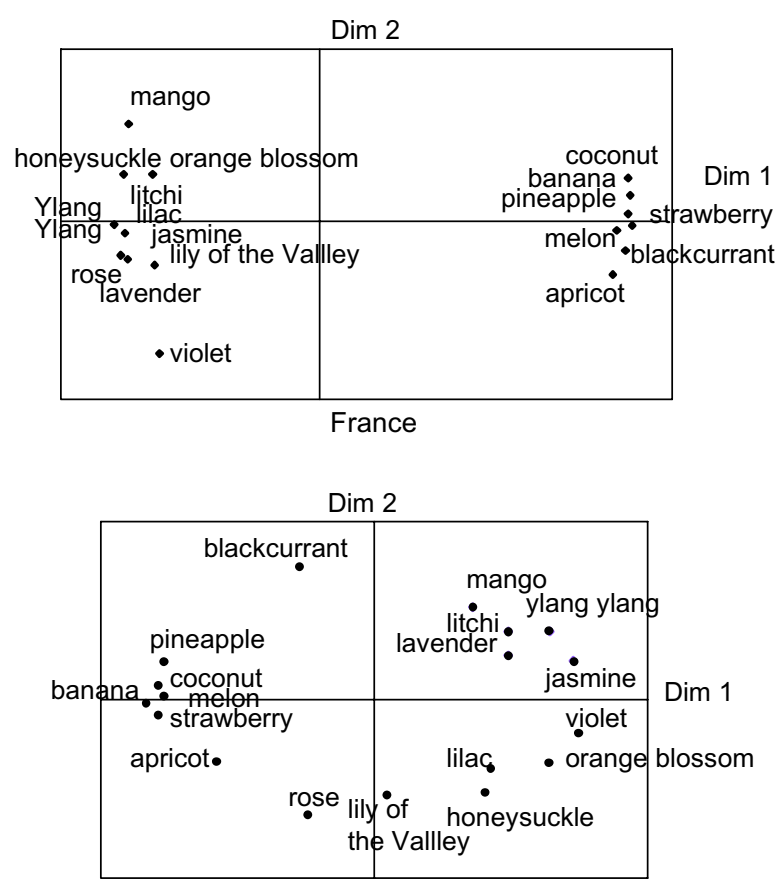

The USA

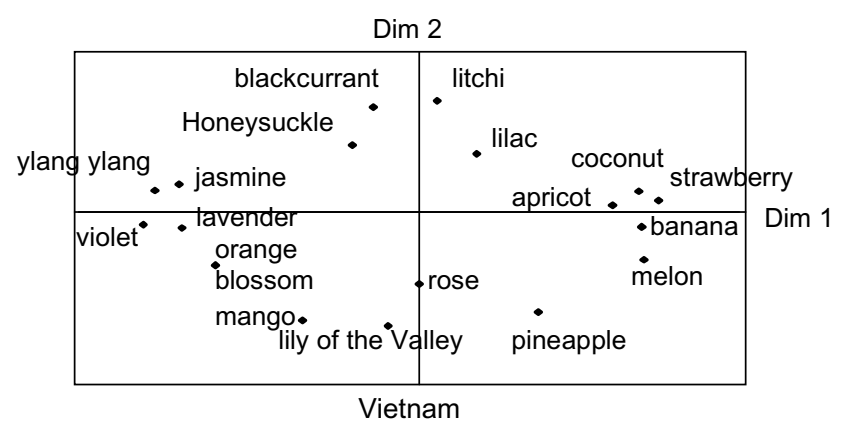

Fig. 4. Two-dimensional solutions from odor sorting made by participants of the three cultures (Dim denotes dimension).

Elmouaffek, \& Sauvegrain (1988) argued that odors are more often sorted on the basis of their common taxonomic origin rather than on the basis of similarities in odor quality. This hypothesis could be confirmed also by this unexpected finding, our French participants separated fruit from flowers odorants very clearly compared to the two other groups of participants whereas it was the opposite trend that emerged from the MDS in the first sorting task. French participants were maybe more accurate in sorting odors according to their identification with a reduced number of homogenous odors rather than with a large set of various odors.

As for the rating task, we found more similarities between French and American participants' odor representations than each of them with Vietnamese participants' representation. This result could be explained by higher similarities between French and American olfactory environments compared to the Vietnamese olfactory environment. This opposition between
Western cultures and Asian cultures has been observed by many authors in chemical senses: in ratings of perceptual dimensions (Ayabe-Kanamura et al., 1998; Pangborn et al., 1988; Wysocki et al., 1991), acceptability of body odors (Schleidt et al., 1981), the use of hedonic scale for food products judgments (Yeh et al., 1998), taste perception, and preferences (Bertino \& Chan, 1986; Yamaguchi, 1991).

\section{Conclusion}

In summary, we found that American, French and Vietnamese participants of our study differed in their judgments for several perceptual dimensions. However, they shared a common general odor representation structured in a few categories, and used the same perceptual dimensions to categorize the odors, such as pleasantness, edibility and cosmetic acceptability. These findings seem to support the claim that some universal cognitive mechanisms might underlie the perception of the world. However, additional studies using different odorants and cultures are needed to verify the generality of this claim.

At a finer level, the consensus between cultures on odor representation stayed consistent only for two of the three groups of participants: the American and French participants' representations. This result may be due to differences in the function attributed to the odors. Indeed, American and French participants described clearly fruit odorants as candies and flower odorants as cosmetics or cleaning products, whereas Vietnamese participants did not use such descriptions. This may come from the fact that, as post-industrial cultures, French and American people are exposed to similar standardized odors from international trades, while Vietnamese people encounter more local aroma which correspond to their own culture (Aubaile Sallenave, 2000). For instance, French and American cultures widely encounter dairy products such as yogurts or ice creams flavored with artificial fruit aroma while it is rarer in Vietnam. Moreover, perfume and cosmetics in general are more frequently used in Western countries than in South-East Asian countries. These examples of differences in food and cosmetic consumptions may influence performances and results in a categorization task such as a sorting task of fruit and flower odorants.

The explanation that Western people and Asian people think differently may also be put forward, although it is difficult to interpret our results in these terms. Some social studies have however revealed differences between Western and Asian cultures in grouping stimuli, judgments of association, judgments of similarity, and category learning (see Nisbett, Peng, Choi, \& Norenzayan (2001), for a review). Western thought is held to be analytic (i.e., focus of attention is 
on objects and their attributes being used to place objects into categories), while Asian thought is held to be holistic (i.e., includes the context in the focus of attention).

\section{Acknowledgements}

Thanks are due to Gail Tillman, Betty Edelman, Nils Penard and two anonymous reviewers for helpful comments on earlier drafts of this paper.

\section{References}

Abdi, H. (1990). Additive-tree representation (with an application to face processing). Lecture Note on Biomathematics, 84, 43-59.

Aubaile Sallenave, F. (2000). Odeurs en alimentation dans quelques sociétés de Méditerranée: premiers résultats d'une enquête sur perception et reconnaissance. Bulletin et Mémoire de la Société d'Anthropologie de Paris, 12, 373-395.

Ayabe-Kanamura, S., Schicker, I., Laska, M., Hudson, R., Distel, H., Kobayakawa, T., \& Saito, S. (1998). Differences in perception of everyday odors: a Japanese-German cross-cultural study. Chemical Senses, 23, 31-38.

Berglund, B., Berglund, U., Engen, T., \& Ekman, G. (1973). Multidimensional analysis of twenty-one odors. Scandinavian Journal of Psychology, 14, 131-137.

Bertino, M., \& Chan, M. M. (1986). Taste perception and diet in individuals with Chinese and Europeans ethnic backgrounds. Chemical Senses, 11, 229-241.

Chastrette, M., Elmouaffek, A., \& Sauvegrain, P. (1988). A multidimensional statistical study of similarities between 74 notes used in perfumery. Chemical Senses, 13, 295-305.

Cohen, J. D., Whinney, B. M., Flatt, M., \& Provost, J. (1993). Psyscope: a new graphique interactive environment for designing psychology experiment. Behavioral Research Methods, Instrument and Computers, 25, 257-271.

Dilks, D. D., Dalton, P., \& Beauchamp, G. K. (1999). Cross-cultural variation in responses in malodors. Chemical Senses, 24, 599.

Distel, H., Ayabe-Kanamura, S., Martinez-Gomez, M., Schicker, I., Kobayakama, T., Saito, S., \& Hudson, R. (1999). Perception of everyday odors-correlation between intensity, familiarity and strength of hedonic judgement. Chemical Senses, 24, 191-199.

Doty, R. L., Applebaum, S., Zusho, H., \& Settle, R. G. (1985). Sex differences in odor identification ability: a cross-cultural analysis. Neuropsychologia, 23(5), 667-672.

Holley, A. (2001). From stimulus reception to category formation in chemical senses. In 4th Pangborn sensory science symposium, Dijon, France.

Hübener, F., Laska, M., Kobayakawa, T., \& Saito, S. (in press). Cross-cultural comparison of olfactory perception in Japanese and Germans. Food Quality and Preference.

Hudson, R., \& Distel, H. (2002). The individuality of odor perception. In C. Rouby, B. Schaal, D. Dubois, R. Gervais, \& A. Holley (Eds.),
Olfaction, taste, and cognition. Cambridge, London: Cambridge Press.

Lawless, H. T. (1989). Exploration of fragrance categories and ambiguous odors using multidimensional scaling and cluster analysis. Chemical Senses, 14, 349-360.

Ly May, H. (2001). Effet de la culture sur la perception, l'identification et l'appréciation des odeurs. Unpublished Mémoire de Diplôme Supérieur d'Etudes et de Recherches, Université de Bourgogne, Dijon, France.

Nisbett, R. E., Peng, K., Choi, I., \& Norenzayan, A. (2001). Culture and systems of thought: holistic versus analytic cognition. Psychological Review, 108, 291-310.

Pangborn, R. M., Guinard, J.-X., \& Davis, R. G. (1988). Regional aroma preferences. Food Quality and Preference, 1, 11-19.

Prescott, J., \& Bell, G. (1995). Cross-cultural determinants of food acceptability: recent research on sensory perceptions and preferences. Trends in Food Science \& Technology, 6, 201-205.

Rabin, M. D., \& Cain, W. S. (1984). Odor recognition: familiarity, identifiability, and encoding consistency. Journal of Experimental Psychology: Learning, Memory, and Cognition, 10, 316-325.

SAS (Ed.). (1989). SAS/STAT ${ }^{\circledR}$ user's guide. Cary, NC: SAS Institute.

Schaal, B., Soussignan, R., Marlier, L., Kontar, F., Karima, I. S., \& Tremblay, R. E. (1997). Variability and invariants in early odours preferences: comparative data from children belonging to three cultures. Chemical Senses, 22, 212.

Schiffman, S. S., Robinson, D. E., \& Erickson, R. P. (1977). Multidimensional scaling of odorants: examination of psychological and physicochemical dimensions. Chemical Senses and Flavor, 2, 375-390.

Schleidt, M., Hold, B., \& Attili, G. (1981). A cross-cultural study on the attitude towards personal odors. Journal of Chemical Ecology, 7, 19-31.

Schleidt, M., Neumann, P., \& Morishita, H. (1988). Pleasure and disgust: memories and associations of pleasant and unpleasant odours in Germany and Japan. Chemical Senses, 13, 279293

Song, H.-J., \& Bell, G. A. (1998). Cross-cultural comparison of judgements of odours by Australians and Indonesians. Chemical Senses, 23, 584-585.

Sugiyama, H., Ayabe-Kanamura, S., \& Kikuchi, T. (2002). Is olfactory mental image sensory-like? Poster presented at the ECRO XIV Biannual Congress of ECRO, Erlangen, Germany.

Ueno, Y. (1993). Cross-cultural study of odor perception in Sherpa and Japanese people. Chemical Senses, 18, 352-353.

Woskow, M. H. (1968). Multidimensional scaling of odors. In N. N. Tanyloc (Ed.), Theories of odors and odor measurement (pp. 147188). Bebek, Istanbul: Robert College Research Center.

Wysocki, C. J., Pierce, J. D., \& Gilbert, A. N. (1991). Geographic, cross-cultural, and individual variation in human olfaction. In $\mathrm{T}$. V. Getchell, R. L. Doty, L. M. Bartoshuck, \& J. B. Snow (Eds.), Smell and taste in health and disease (pp. 287-314). New York: Raven Press.

Yamaguchi, S. (1991). Basic properties of umami and effects on humans. Physiology \& Behavior, 49, 833-841.

Yeh, L. L., Kim, K. O., Chompreeda, P., Rimkeeree, H., Yau, N. J. N., \& Lundahl, D. S. (1998). Comparison in use of the 9-point hedonic scale between Americans, Chinese, Koreans, and Thai. Food Quality and Preference, 9, 413-419. 